\title{
Synchronous Ventral Hernia Repair in Bariatric Patients
}

\author{
Daniel L. Chan • Michael L. Talbot
}

Published online: 2 April 2014

(C) Springer Science+Business Media New York 2014

\section{Dear Editor,}

We note with interest the article of Eid et al. [1] and correspondence in the journal recently on the topic issue of timing of ventral hernia in bariatric patients. We note that this correspondence recommends a range of options in managing these patients but provides little evidence to back up these recommendations. While the issue of ventral hernia is obviously complex, we are concerned that recommending non-standard treatments may lead to outcomes that expose patients to increased risks and repeated surgical procedures. The use of suture and absorbable mesh repairs have been shown to have high recurrence rates, and leaving hernias untreated will expose patients to the certain requirement for later surgery and the risks of small bowel obstruction associated with their rapid weight loss.

With this consideration, we consider all patients undergoing bariatric procedures with ventral hernias as candidates for synchronous repair, unless they had massive abdominal wall defects or cutaneous redundancy, in which case they are usually offered sleeve gastrectomy followed by delayed abdominal wall reconstruction. This number is small.

We have attempted to answer the question of whether patients having bariatric surgery can have simultaneous ventral hernia repair if it is felt that the defect is amenable to laparoscopic repair by studying our outcomes [2]. In our series of 45 patients undergoing synchronous bariatric surgery and ventral hernia repair, the results were similar to those of laparoscopic repair alone. Two patients developed

D. L. Chan $\cdot$ M. L. Talbot $(\bowtie)$

Upper Gastrointestinal Surgery Unit, St. George Hospital, Kogarah, NSW 2217, Australia

e-mail: m.talbot@unsw.edu.au

D. L. Chan $\cdot$ M. L. Talbot

Department of Surgery and the St George Clinical School, University

of New South Wales, High St., Kensington, NSW 2052, Australia infected seromas that responded to simple drainage, and there have been no mesh removals or clinical hernia recurrence to date. This fits with the results of a Cochrane review comparing open and laparoscopic techniques concluding that laparoscopic hernia repair infection rates were $3.14 \%$ (range 0-6.06\%) [3].

While valid concerns exist about the potential complication rate of using permanent mesh in patients having bariatric surgery, the rate of complications appear reassuringly low. Surgeons can be comfortable making decisions based on data, and in this situation, these data suggest that patients presenting for bariatric surgery with concomitant hernia can be offered standard treatment with the expectation of standard outcomes. The outcomes of non-standardised techniques such as suture repair or using biologics are poorly known, and since these repairs are either more likely to fail or are more complex than usual laparoscopic repair methods, they are hard to recommend in the face of established safe and effective techniques.

In the future, a multicentre study may be able to provide sufficient numbers for a case-control or randomised trial, but until this occurs, clinicians must incorporate current evidence through available case-series, experience and extrapolation from published trials of established techniques.

Conflict of Interest No conflicts of interest declared.

\section{References}

1. Eid GM, Wikiel KJ, Entabi F, et al. Ventral hernias in morbidily obese patients: a suggested algorithm for operative repair. Obes Surg. 2013;23(5):703-9.

2. Chan DL, Talbot ML, Chen Z, et al. Simultaneous ventral hernia repair in bariatric surgery. ANZ J Surg. 2013. doi:10.1111/ans.12174.

3. Sauerland S, Walgenbach M, Habermalz B, et al. Laparoscopic versus open surgical techniques for ventral or incisional hernia repair. Cochrane Database Syst Rev. 2011;16(3), CD007781. 